\title{
Carcinoma renal que debuta como cuadro neurológico paraneoplásico
}

\author{
Hens $\mathrm{MJ}^{*}$, Camacho B*, Maestre A*, González V*, Villegas I*, Magaña M**. \\ *Servicios de Neurología y **Urología. Complejo Hospitalario de Jaén.
}

Actas Urol Esp. 2008;32(6):645-648

\begin{abstract}
RESUMEN
CARCINOMA RENAL QUE DEBUTA COMO CUADRO NEUROLÓGICO PARANEOPLÁSICO

Introducción: Los procesos tumorales pueden de manera ocasional mostrar su primera manifestación con una sintomatología neurológica, no originada por metástasis, sino del denominado tipo paraneoplásico, que puede aparecer incluso años antes de los síntomas habituales del tumor. Este tipo de manifestaciones iniciales pueden ser la clave del descubrimiento de una neoplasia oculta.

Métodos: Presentamos el caso de un varón de 66 años afecto de un carcinoma renal, cuya manifestación inicial consistió únicamente en un cuadro neurológico progresivo de tipo cerebeloso paraneoplásico, aparecido 6 años antes de la primera sintomatología urológica.

Conclusiones: La aparición de una sintomatología neurológica cerebelosa progresiva puede ser la única y primera manifestación de un carcinoma renal, incluso años antes de las primeras manifestaciones urológicas. Debe sospecharse la existencia de una neoplasia oculta en los pacientes con esta sintomatología.

Palabras clave: Carcinoma de Células Renales. Cerebelo. Enfermedades cerebelosas. Degeneración cerebelar paraneoplásica. Ataxia.

\section{ABSTRACT}

RENAL CELL CARCINOMA PRESENTING AS PARANEOPLASTIC NEUROLOGICAL DYSFUNCTION

Introduction: Malignant tumors sometimes initiate as paraneoplastic syndromes even years before the most common symptoms appear. These first manifestations could be the key for the diagnosis of "occult" malignancy.

Methods: We report the case of a 66 year old man with a renal cell carcinoma. The first symptom was a paraneoplastic cerebellar degeneration appeared 6 years before the first urologic manifestations.

Conclusions: A progressive cerebellar syndrome could be the first manifestation of a renal cell carcinoma, even years before the first urologic symptoms. We must suspect an occult neoplasia in such patients.

Keywords: Carcinoma, Renal cell. Cerebellum. Cerebellar diseases. Paraneoplastic cerebellar degeneration. Ataxia.
\end{abstract}

$\mathrm{L}$ os procesos tumorales pueden ocasionalmente mostrar su primera manifestación con una sintomatología neurológica, no originada por invasión local ni por metástasis, sino del denominado tipo paraneoplásico, que puede aparecer incluso años antes de los sintomas habituales del tumor ${ }^{1}$. Estas manifestaciones iniciales pueden ser la clave del descubrimiento de una neoplasia oculta. Presentamos el caso de un paciente afecto de un carcinoma renal cuya manifestación clínica inicial consistió únicamente en un síndrome cerebeloso de tipo paraneoplásico, y que fue la sintomatología que finalmente condujo a la detección del tumor.

\section{CASO CLÍNICO}

El paciente, varón de 66 años, venía sufriendo un trastorno de la marcha caracterizado por inestabilidad y ataxia, de aproximadamente 6 años de evolución, de carácter progresivo. Contaba con antecedentes de HTA y síndrome vertiginoso periférico. No existían antecedentes de otras patologías que pudiesen justificar sus síntomas, ni pudieron ser atribuidas a una causa etílico-carencial, pues su dieta era adecuada y era abstemio desde años antes de aparecer su sintomatología. Su examen neurológico mostró una marcha muy inestable de tipo atáxico, con base de sustentación ampliada, marcha en 
tandem muy dificultosa, y signo de Babinski bilateral, sin dismetría, nistagmo ni anomalías sensitivas, incluyendo la sensibilidad propioceptiva. No mostraba signos de parkinsonismo, papiledema ni otras anomalías exploratorias. La resonancia magnética cerebral mostró atrofia cerebral y especialmente cerebelosa, sin tumor primario, metástasis ni otras anomalías. Se descartaron las causas más frecuentes de su sintomatología, mediante analítica general, hormonas tiroideas, vitamina $\mathrm{B}_{12}$, ácido fólico, serología a lúes, Brucella y Borrelia, curva de glucemia, espectro electroforético e inmunoglobulinas en sangre. Considerando un posible origen paraneoplásico de sus síntomas, se efectuaron pruebas para intentar descubrir una posible neoplasia oculta, siendo normales las determinaciones de PSA, CEA, $\alpha$-fetoproteína, $\beta$-HCG, $\beta$-2-microglobulina, Ca-199 , anticuerpos anti-Yo y anti-HU en suero. La radiografía simple de abdomen no mostró anomalías destacables, si bien las siluetas renales resultaban sólo parcialmente visibles. Una ecografía abdominal mostró un riñón derecho con contornos lobulados, apareciendo una masa sólida renal derecha, sugerente de neoplasia. A raíz de dicho hallazgo se solicitó una urografía intravenosa, que mostró un riñón pequeño, con disminución de la distancia córticopapilar, con signos de pielonefritis evolucionada, sin otras anomalías. La TAC abdominal mostró un gran tumor renal derecho, que incidía en la pelvis renal, sin adenopatías o afectación de otros órganos. Súbitamente, y antes de que pudiera realizarse la extirpación del tumor, apareció una intensa hematuria, como primera y única manifestación urológica del proceso, procediéndose a la nefrectomía radical derecha con adrenalectomía. El estudio histológico mostró un carcinoma de células renales, de patrón predominante oncocítico con grado de diferenciación GIII, con invasión de la vena renal, y signos de pielonefritis crónica peritumoral, linfadenitis reactiva inespecífica sin evidencia de metástasis ganglionares. Tras descartarse metástasis mediante el pertinente estudio de extensión, se estableció la estadificación clínica T3 NO MO, G III. A partir de la extirpación del tumor, el cuadro neurológico se estabilizó, desapareciendo el deterioro progresivo de la deambulación que hasta entonces había experimentado. A lo largo de 8 años de seguimiento no han aparecido metástasis, el cuadro cerebeloso se halla estabilizado sin empeoramiento, ni ha aparecido nueva sintomatología neurológica, confirmándose de este modo que su sintomatología estaba originada por un síndrome paraneoplásico causado por el carcinoma renal.

\section{DISCUSIÓN}

Los procesos tumorales pueden ocasionalmente mostrar su primera manifestación con una sintomatología neurológica, que puede ser debida a invasión local, metástasis, o del denominado tipo paraneoplásico. Un $11 \%$ de las metástasis de los carcinomas de células renales tienen su asiento en el sistema nervioso central, siendo la afectación metastásica solitaria del sistema nervioso central muy infrecuente $^{2-5}$. Sin embargo, en nuestro caso la afectación neurológica del paciente no fue debida a metástasis, sino a un síndrome paraneoplásico, aparecido años antes de los síntomas habituales del tumor, como ha sido previamente descrito ${ }^{1}$.

Los sindromes paraneoplásicos consisten en la afectación de órganos o tejidos alejados del tumor primario o sus metástasis, y pueden afectar a la mayoría de órganos y tejidos del organismo. La mayoría de estos cuadros se producen por mecanismos inmunológicos, que producen una degeneración autoinmune del sistema nervioso ${ }^{1}$. Al igual que en el caso que presentamos, cuando aparece el síndrome paraneoplásico el tumor puede ser aún asintomático, y los sintomas neurológicos pueden preceder al diagnóstico del tumor, hasta el punto que es esta sintomatología neurológica la que lleva a consultar, y puede constituir la clave para el diagnóstico del tumor oculto ${ }^{6,7}$.

Casi todos los tumores primarios pueden causar síndromes paraneoplásicos, siendo los más frecuentes el cáncer pulmonar de células pequeñas, el de mama, los ginecológicos y los linfomas ${ }^{8}$. Estos mismos tumores, especialmente el de ovario, son los que con más frecuencia se asocian a degeneración cerebelosa paraneoplásica ${ }^{9}$. En lo que respecta al carcinoma de células renales, se ha estimado que aproximadamente un tercio de los pacientes afectados muestra síntomas y signos de un síndrome paraneoplásico, abarcando desde síntomas constitucionales (fiebre, pérdida de peso) hasta trastornos específicos metabólicos y bioquímicos (hipercalcemia, hipertensión, policitemia, hepatopatía no metastásica, amiloidosis, galactorrea, síndrome de Cushing, hiper e hipoglucemia, etc), por lo que clá- 
sicamente este tumor llegó a ser denominado "el gran enmascarado de la medicina”, dada su capacidad de afectar a casi todos los órganos y sistemas ${ }^{10}$. En lo referente a la afectación neurológica, los síndromes paraneoplásicos asociados al carcinoma renal pueden incluir manifestaciones muy diversas, que incluyen tanto al sistema nervioso central como al periférico. Han sido descritas polineuroptías sensitivas y motoras, incluso con parálisis bilateral del nervio frénico, esclerosis lateral amiotrófica, degeneración cerebelosa subaguda, síndrome del lóbulo frontal, cuadros extrapiramidales de tipo coréico y balístico, y síndrome de opsoclonus-mioclonus ${ }^{10-19}$.

La asociación de un síndrome paraneoplásico con un carcinoma renal puede proporcionar varios aspectos de interés práctico. Su presencia no representa un marcador de la existencia de metástasis, ni indica un peor pronóstico, pero si en algún momento se observa una recurrencia del síndrome paraneoplásico previo, debe poner en alerta de una posible progresión del proceso tumoral ${ }^{10}$. En otras ocasiones, como en el caso que presentamos, la aparición del síndrome paraneoplásico puede ser la clave que inicie la búsqueda de un tumor oculto. Sin embargo, hasta en el $20 \%$ de los casos de pacientes con síndrome paraneoplásico no es posible confirmar la presencia de un tumor, incluso tras la necropsia ${ }^{8}$.

Resulta infrecuente que un carcinoma renal se asocie, como en el caso que presentamos, a un síndrome cerebeloso paraneoplásico. El carcinoma ovárico es el que con más frecuencia e asocia a esta sintomatología, seguido por el de mama, pulmón (especialmente el de células pequeñas), y la enfermedad de Hodgkin ${ }^{9}$. No obstante, como en nuestro caso, la aparición de una sintomatología neurológica cerebelosa progresiva puede ser la única y primera manifestación de un carcinoma renal, incluso años antes de las primeras manifestaciones urológicas.

La forma de presentación clínica del carcinoma renal puede ser diversa, pudiendo permanecer silente y ser descubierto de forma casual, producir alteraciones por el propio proceso expansivo renal o debutar con las manifestaciones clínicas derivadas de la presencia de metástasis o síndromes paraneoplásicos, éstos últimos presentes en más del $20 \%$ de los carcinomas renales, siendo en algunos casos su manifestación inicial ${ }^{20}$. Esta situación fue la que condujo al estudio y posterior diagnóstico de nuestro paciente.
La patogénesis de estos sindromes paraneoplásicos consiste en la liberación por las células tumorales de sustancias con acción endocrino-metabólica o bien que inducen la liberación de mediadores de la inflamación, principalmente citoquinas e interleucinas. Se ha conseguido identificar a la IL-6 como responsable de algunos de estos procesos ${ }^{20}$.

El proceso diagnóstico del carcinoma renal ha variado notablemente en los últimos años, siendo cada vez más frecuente el diagnóstico incidental durante exploraciones complementarias por enfermedades no relacionadas o ecografías realizadas como chequeo ${ }^{20}$. El caso que presentamos no puede considerarse "incidental" en estricto sentido, pues el paciente ya mostraba manifestaciones neurológicas paraneoplásicas, que motivaron su estudio rastreando un tumor primario oculto. En todo caso, la larga supervivencia de nuestro paciente, que a lo largo de 8 años de seguimiento no ha desarrollado metástasis, se asimila al mejor pronóstico observado en los casos detectado de forma incidental ${ }^{21,22}$.

\section{CONCLUSIÓN}

Una sintomatología neurológica progresiva de tipo cerebeloso puede ser la primera manifestación de una neoplasia oculta, entre las que puede encontrarse el carcinoma renal.

\section{REFERENCIAS}

1. Darnell RB, Posner JB. Paraneoplastic Syndromes Involving the Nervous System. N Engl J Med. 2003;349(16):1543-1554.

2. Dekernion JB, Ramming RP, Smith RB. The natural history of metastatic renal cell carcinoma: a computer analysis. J Urol. 1978(2);120:148-152.

3. Saitoh H. Distant metastasis of renal adenocarcinoma. Cancer. 1981;48(6): 1487-1491

4. Pereira Arias JG, Ullate Jaime V, Valcárcel Martín F, Onaniel Perez VJ, Gutierrez Díez JM, Ateca Díaz-Obregon R, et al. Epístaxis como primera manifestación de un adenocarcinoma renal diseminado. Actas Urol Esp. 2002;26(5):361-365.

5. Herrera Puerto J, Soler Fernández J, Asuar Aydillo S, Domínguez Bravo C, Cabezudo Alzar J. Metástasis cerebral solitaria de un carcinoma de células renales. Actas Urol Esp. 2003;27(6):468-470.

6. Peterson K, Rosenblum MK, Kotanides H, Posner JB. Paraneoplastic cerebellar degeneration. I. A clinical analysis of 55 antiYo antibody positive patients. Neurology. 1992;42(10):19311937.

7. Graus F, Keime-Guibert F, Reñe R, Benyahia B, Ribalta T, Ascaso C, et al. Anti-Hu-associated paraneoplastic encephalomyelitis: analysis of 200 patients. Brain. 2001;124(6):1138-1148.

8. Rees JH. Paraneoplastic syndromes: when to suspect, how to confirm, and how to manage. Journal Neurol Neurosurg Psychiatry. 2004;75 Suppl 2:ii43-50. 
9. Graus F. Síndromes neurológicos paraneoplásicos. En: Tratado de Neurología. Codina Puiggrós A, Ed. ELA. Madrid. 1994;543549 .

10. Palapattu GS, Kristo B, Rajfer J. Paraneoplastic Syndromes in Urologic Malignancy: The Many Faces of Renal Cell Carcinoma. Rev Urol. 2002;4(4):163-170.

11. Gimeno Campos MJ, Sanchis Minguez C, Diez de Diego P, Sanchez Villasante J. Opsoclonus-myoclonus: paraneoplastic syndrome associated with renal adenocarcinoma. Rev Esp Anestesiol Reanim. 2006;53(1):54-55.

12. Evans BK, Fagan C, Arnold T, Dropcho EJ, Oh SJ. Paraneoplastic motor neuron disease and renal cell carcinoma: improvement after nephrectomy. Neurology. 1990;40(6):960962.

13. De Luca S, Terrone C, Crivellaro S, De Zan A, Polo P, Vigliani $\mathrm{MC}$, et al. Opsoclonus-Myoclonus Syndrome as a Paraneoplastic Manifestation of Renal Cell Carcinoma. A Case Report and Review of the Literature. Urol Int. 2002;68(3):206-208.

14. Thomas NE, Passamonte PM, Sunderrajan EV, Andelin JB, Ansbacher LE. Bilateral diaphragmatic paralysis as a possible paraneoplastic syndrome from renal cell carcinoma. Am Rev Respir Dis. 1984;129(3):507-509.

15. Kujawa KA, Niemi VR, Tomasi MA, Mayer NW, Cochran E, Goetz CG. Ballistic-Choreic Movements as the Presenting Feature of Renal Cancer. Arch Neurol. 2001;58(7):1133-1135.

16. Rijnders B, Decramer M. Reversibility of paraneoplastic bilateral diaphragmatic paralysis after nephrectomy for renal cell carcinoma. Ann Oncol. 2000;11(2):221-225.

17. Hagel C, Stavrou D, Hansen HC. Paraneoplastic frontal lobe disorder and ataxia in renal cell carcinoma. Neuropathol Appl Neurobiol. 2005;31(1):97-99.
18. Rubio Nazábal E, Marey López J, Alvarez Pérez P, López Facal S, Alonso Magdalena L. Síndrome opsoclonus-mioclonus en paciente con cáncer de ovario. An Med Interna (Madrid). 2003;20(7):42-44

19. Martin JC, Lara M, Alvarez Ferreira J, Frank A, Díez Tejedor E. Subacute paraneoplastic cerebellar degeneration in a patient with renal adenocarcinoma. Rev Clin Esp. 1998;198(4):259260.

20. Sánchez Zalabardo D, López Ferrandis J, Arocena GarcíaTapia J, Rogojo Balboa M, Fernández Montero JM, Rosell Costa $\mathrm{D}$, et al. Clínica, diagnóstico y pronóstico del carcinoma renal. Actas Urol Esp. 2002;26(8):532-540.

21. Congregado Ruiz B, Medina López RA, Sánchez Gómez E, Morales López A, Pascual del Pobil JL. Diagnóstico incidental del carcinoma renal. ¿Implica un mejor pronóstico?. Actas Urol Esp. 2001;25(4):278-282

22. Ortiz Gorraiz M, Vicente Prados FJ, Rosales Leal JL, Honrubia Vílchez B, Martínez Morcillo A, Cózar Olmo JM et al. Valoración de factores pronósticos de la supervivencia en una serie de 202 pacientes intervenidos por carcinoma de células renales. Actas Urol Esp. 2005;29(2):179-189.

Correspondencia autor: Dr. M.J. Hens

Servicio de Neurología. Complejo Hospitalario de Jaén

Avda. del Ejército Español, 10 - 23007 Jaén

Tel.: 953008579

E-mail autor: med000143@saludalia.com

Información artículo: Nota Clínica

Trabajo recibido: diciembre 2006

Trabajo aceptado: enero 2007 